Bull. Austral. Math. Soc.

$11 \mathrm{R} 58,11 \mathrm{R} 60$

VOL. 65 (2002) [385-392]

\title{
MAXIMAL INDEPENDENT SYSTEM OF UNITS IN FUNCTION FIELDS
}

\author{
HWANyup Jung AND JaEhyUn AhN
}

\begin{abstract}
In this paper, we construct a new maximal independent system of units in cyclotomic function fields and their subfields. We also calculate its index in the full units group and show that it is smaller than the index of Feng-Yin's system.
\end{abstract}

\section{INTRODUCTION}

In this paper we study maximal independent systems of units in cyclotomic function fields and their subfields. In the classical case, Ramachandra [7] was the first to study such systems and his result was improved by Levesque [6]. Recently Greither [3] invent a general machinery to construct a maximal independent system of units and also found a system which has smaller index in the full unit group than Ramachandra's and Levesque's one. Greither's result is generalised to arbitrary Abelian number fields by Kucera [5]. In the function field case, such systems are studied by Feng and Yin [2]. Their result may be regarded as an analog of Ramachandra and Levesque (even if they worked in any subfields of cyclotomic function fields). Adapting ideas of Greither and Kucera, we construct a new maximal independent system of units in cyclotomic function fields and their subfields. Our system has smaller index in the full unit group than Feng-Yin's one.

The layout of this paper is as follows. In Section 2, we give the notation for cyclotomic function fields and their subfields, and preliminary results needed in this paper. In Section 3, we generalise Greither's general machinery of constructing a maximal independent system of units to any subfields of cyclotomic function fields and obtain a formula for the index (Theorem 3.1 and Theorem 3.2). In Section 4, we introduce $\mathcal{C}_{\beta}$ a new maximal independent system of units and calculate its index in the full units group (Theorem 4.1). In real extension, we also define another system $\mathcal{C}_{\beta}^{\prime}$ which has smaller index than $\mathcal{C}_{\beta}$ (Corollary 4.2). In Section 5, we give a numerical comparison of the index $i_{\mathcal{B}}$ and Feng-Yin's index $i(\mathcal{D})$. Finally in Section 6, we construct an $R$-cyclic submodule of the Sinnott's module $U$ and calculate its index.

Received 26th July, 2001

The first author is supported in part by BK21 Project at Korea University.

Copyright Clearance Centre, Inc. Serial-fee code: 0004-9727/02 \$A2.00+0.00. 


\section{BASIC FACTS AND NOTATIONS}

Let $\mathbb{A}=\mathbb{F}_{q}[T]$ be the ring of polynomials over a finite field $\mathbb{F}_{q}$ with $q$ elements, and $k=\mathbb{F}_{q}(T)$. For each monic polynomial $M \in \mathbb{A}$, one uses the Carlitz module $\rho$ to construct a field extension $K_{M}$, called the $M$-th cyclotomic function field. Let $\infty$ be the place of $k$ associated to $(1 / T)$ and $k_{\infty}$ be the completion of $k$ at $\infty$. We fix a primitive $M$-torsion point $\lambda_{M} \in K_{M}$ of the Carlitz module. It is well known that there is an isomorphism $\Psi:(\mathbb{A} / M \mathbb{A})^{*} \rightarrow \operatorname{Gal}\left(K_{M} / k\right), B \bmod M \mapsto \sigma_{B}$, with $\sigma_{B}\left(\lambda_{M}\right)=\rho_{B}\left(\lambda_{M}\right)$. We write $\lambda_{M}^{B}=\rho_{B}\left(\lambda_{M}\right)$ for simplicity. It is also known that $J=\Psi\left(\mathbb{F}_{q}^{*}\right)$ is the decomposition group and the inertia group of $\infty$ in $K_{M}$. Let $K_{M}^{+}$be the maximal real subfield of $K_{M}$, that is, the fixed field of $J$.

Let $F$ be a subfield of some cyclotomic function field with conductor $M$, that is, $K_{M}$ is the smallest cyclotomic function field containing $F$. Let $G=\operatorname{Gal}(F / k)$ and $R=\mathbb{Z}[G]$ the integral group ring. Let $F^{+}$be the maximal real subfield of $F, J_{F}=\operatorname{Gal}\left(F / F^{+}\right)$ and $\delta_{F} \doteq\left|J_{F}\right|$. We say $F$ is a real extension if $J_{F}$ is trivial. We recall the definition of cyclotomic numbers and cyclotomic units $([4$, Section 3]). For a monic $N$ in $\mathbb{A}$, let $F_{N}=K_{N} \cap F$ and $\lambda_{N}$ be a primitive $N$-torsion point. Let $\mathcal{D}_{F}$ be the subgroup of $F^{*}$ generated by $\mathbb{F}_{q}^{*}$ and all elements $N_{K_{N} / F_{N}}\left(\lambda_{N}^{A}\right)$ and $\mathcal{C}_{F}=\mathcal{D}_{F} \cap \mathcal{O}_{F}^{*}$, where $\mathcal{O}_{F}$ is the integral closure of $\mathbb{A}$ in $F$ and $\mathcal{O}_{F}^{*}$ is the unit group of $\mathcal{O}_{F}$. For any $X \subset G$, let $s(X)=\sum_{\sigma \in X} \sigma \in R$. We factor $M$ as $M=\prod_{i=1}^{s} P_{i}^{e_{i}}$, where $P_{i}$ is a monic irreducible in $\mathbb{A}$ and let $M_{0}^{\sigma \in X}=\prod_{i=1}^{s} P_{i}$. Let $S=\{1, \ldots, s\}$ and $\mathbb{P}_{S}$ be the set of all proper subsets of $S$. For each $i \in S$, we let $T_{i}$ and $D_{i}$ be the inertia group and the decomposition group of $P_{i}$ in $F$ respectively. And let $t_{i}=\left|T_{i}\right|\left(f_{i}=\left|D_{i}\right| /\left|T_{i}\right|\right.$, and $g_{i}=|G| /\left|D_{i}\right|$ respectively) denote the ramification degree (inertia, and decomposition degree respectively) of $P_{i}$ in $F$. We fix a Frobenius automorphism $\mathcal{F}_{i} \in G$ of $P_{i}$, which is well-defined modulo $T_{i}$ and denote $\nu_{i}=\sum_{j=1}^{f_{i}} \mathcal{F}_{i}^{j} \in R$. For each subset $I$ of $S$, we also introduce following notations; $M_{I}=\prod_{i \in I} P_{i}^{e_{i}}, T_{I}=\prod_{i \in I} T_{i}$, $D_{I}=\prod_{i \in I} D_{i}, \nu_{I}=\prod_{i \in I} \nu_{i}$, and $n_{I}=\left(\prod_{i \in I} t_{i}\right) /\left|T_{I}\right|$. Let $\widehat{G}=\operatorname{Hom}\left(G, \mathbb{C}^{*}\right)$ be the character group of $G$. A character $\chi$ is called even if $\chi$ is trivial on $J_{F}$ and called odd otherwise. For $\chi \in \widehat{G}, \chi$ can be regarded as a map from $\mathbb{A}$ to $\mathbb{C}$ by Artin map. Then

$$
\chi\left(P_{i}\right)= \begin{cases}\chi\left(\mathcal{F}_{i}\right) & \text { if } T_{i} \subset \operatorname{ker} \chi \\ 0 & \text { if } T_{i} \not \subset \operatorname{ker} \chi\end{cases}
$$

As in the classical case the conductor of a character $\chi$ is defined and we denote by $F_{\chi}$ the conductor of $\chi$.

\section{THE GENERAL MACHINERY}

In this section, we extend Greither's general machinery of constructing a maximal independent system of units to any subfields of cyclotomic function fields. For $I \in \mathbb{P}_{S}$, we 
put $\lambda_{I}=N_{K_{M / M_{I}} / F_{M / M_{I}}}\left(\lambda_{M / M_{I}}\right)$. We consider functions $\beta: \mathbb{P}_{S} \rightarrow \mathbb{Z}[G]$. A function $\beta$ is called multiplicative if $\beta(\emptyset)=1$, and $\beta(I \cup J)=\beta(I) \beta(J)$ whenever both sides are defined and the intersection $I \cap J$ is empty. Clearly, a multiplicative function $\beta$ is determined by the values $\beta(\{i\})$ and these can be assigned arbitrarily. We denote $\beta(\{i\})$ by $\beta(i)$ for simplicity. For any $x \in F$ and $\alpha \in R$, one has a well-defined power $x^{\alpha}$. Thus we may define

$$
\lambda(\beta)=\prod_{I \in \mathbb{P}_{S}} \lambda_{I}^{n_{I} \beta(I)} \in \mathcal{D}_{F}
$$

Let $\mathcal{R}$ be a full set of representatives for $G / J_{F}$ containing 1 and let $\mathcal{R}^{*}=\{\sigma \in \mathcal{R}: \sigma \neq 1\}$. Let $\mathcal{C}_{\beta}$ be the group generated by $\mathbb{F}_{q}^{*}$ and $\left\{\lambda(\beta)^{\sigma-1} ; \sigma \in \mathcal{R}^{*}\right\}$.

THEOREM 3.1. Let $h\left(\mathcal{O}_{F^{+}}\right)$be the ideal class number of $\mathcal{O}_{F^{+}}$and $Q_{0}=\left[\mathcal{O}_{F}^{*}\right.$ : $\left.\mathcal{O}_{F^{+}}^{*}\right]$. For any function $\beta: \mathbb{P}_{S} \rightarrow \mathbb{Z}[G]$, we have

$$
\left[\mathcal{O}_{F}^{*}: \mathcal{C}_{\beta}\right]=Q_{0}\left((q-1) / \delta_{F}\right)^{\left[F^{+}: k\right]-1} h\left(\mathcal{O}_{F^{+}}\right) i_{\beta}
$$

where

$$
i_{\beta}=\left|\prod_{\chi \neq 1, e v e n}\left(\sum_{I \in \mathbb{P}_{S},\left(F_{\chi}, M_{I}\right)=1} n_{I}\left|T_{I}\right| \chi(\beta(I)) \prod_{i \notin I}\left(1-\bar{\chi}\left(P_{i}\right)\right)\right)\right| .
$$

Moreover $i_{\beta}=0$ means that the index of $\mathcal{C}_{\beta}$ in $\mathcal{O}_{F}^{*}$ is infinite.

Proof: The logarithm map $l: F^{*} \rightarrow \mathbb{Q}[G]$ is defined by $l(x)=\sum_{\sigma \in G} v_{\infty}\left(x^{\sigma}\right) \sigma^{-1}$, where $v_{\infty}$ is the normalised valuation at $\infty$. Since $\operatorname{ker} l \cap \mathcal{O}_{F}^{*}=\operatorname{ker} l \cap \mathcal{C}_{\beta}=\mathbb{F}_{q}^{*}$, we have

$$
\begin{aligned}
{\left[\mathcal{O}_{F}^{*}: \mathcal{C}_{\beta}\right] } & =\left[l\left(\mathcal{O}_{F}^{*}\right): l\left(\mathcal{C}_{\beta}\right)\right] \\
& =\left(l\left(\mathcal{O}_{F}^{*}\right): e^{+} R_{0}\right)\left(e^{+} R_{0}: l\left(\mathcal{C}_{\beta}\right)\right) \\
& =\frac{\delta_{F}^{1-\left[F^{+}: k\right]} Q_{0}}{R\left(F^{+}\right)}\left(e^{+} R_{0}: l\left(\mathcal{C}_{\beta}\right)\right),
\end{aligned}
$$

where $e^{+}=s\left(J_{F}\right) / \delta_{F}, R\left(F^{+}\right)$the regulator of $F^{+}$and $R_{0}$ is the augmentation ideal of $R$. The last equality follows from [1, equation (3.8)]. Now we consider the transition matrix of the generators $\left\{l\left(\lambda(\beta)^{\sigma-1}\right): \sigma \in \mathcal{R}^{*}\right\}$ of $l\left(\mathcal{C}_{\beta}\right)$ with respect to the basis $\left\{e^{+}\left(\sigma^{-1}-1\right)\right.$ : $\left.\sigma \in \mathcal{R}^{*}\right\}$ of $e^{+} R_{0}$. Since $\lambda(\beta)^{\sigma-1}$ is unit and $J_{F}$ is the inertia group of $\infty$, we have

$$
\begin{aligned}
l\left(\lambda(\beta)^{\sigma-1}\right) & =\sum_{\tau \in G} v_{\infty}\left(\lambda(\beta)^{(\sigma-1) \tau}\right) \tau^{-1} \\
& =\sum_{\tau \in \mathcal{R}} v_{\infty}\left(\lambda(\beta)^{(\sigma-1) \tau}\right) s\left(J_{F}\right) \tau^{-1} \\
& =\sum_{\tau \in \mathcal{R}^{*}} \delta_{F} v_{\infty}\left(\lambda(\beta)^{(\sigma-1) \tau}\right) e^{+}\left(\tau^{-1}-1\right)
\end{aligned}
$$


Thus by the Dedekind determinant formula (see [8, Lemma 5.26]), we get

$$
\begin{aligned}
\left(e^{+} R_{0}: l\left(\mathcal{C}_{\beta}\right)\right) & =\left|\prod_{\chi \neq 1, \text { even }} \sum_{\sigma \in \mathcal{R}} \bar{\chi}(\sigma) \delta_{F} v_{\infty}\left(\lambda(\beta)^{\sigma}\right)\right| \\
& =\left|\prod_{\chi \neq 1, \text { even }} \sum_{\sigma \in G} \bar{\chi}(\sigma) v_{\infty}\left(\lambda(\beta)^{\sigma}\right)\right| \\
& =\left|\prod_{\chi \neq 1, \text { even }} \sum_{I \in \mathbb{P}_{S}} \sum_{\sigma \in G} \bar{\chi}(\sigma) v_{\infty}\left(\lambda_{I}^{\sigma n_{I} \beta(I)}\right)\right|
\end{aligned}
$$

For $\chi \neq 1$, even and $I \in \mathbb{P}_{S}$, by $[\mathbf{1}$, equation (3.5)], we get

$$
\begin{aligned}
\sum_{\sigma \in G} \bar{\chi}(\sigma) v_{\infty}\left(\lambda_{I}^{\sigma n_{I} \beta(I)}\right) & =\chi(\beta(I)) \sum_{\sigma \in G} \bar{\chi}(\sigma) v_{\infty}\left(\lambda_{I}^{\sigma n_{I}}\right) \\
& =\chi(\beta(I)) \chi\left(l\left(N_{K_{M / M_{I}} / F_{M / M_{I}}}\left(\lambda_{M / M_{I}}\right)^{n_{I}}\right)\right) \\
& =\chi(\beta(I)) n_{I}(q-1) L_{k}(0, \bar{\chi}) \chi\left(s\left(T_{I}\right)\right) \prod_{i \notin I}\left(1-\bar{\chi}\left(P_{i}\right)\right),
\end{aligned}
$$

where $L_{k}(0, \bar{\chi})$ is the Artin $L$-function associated with character $\bar{\chi}$.

If $F_{\chi} \nmid M / M_{I}$ (that is, $T_{I} \not \subset \operatorname{ker} \chi$ ), then $\chi\left(s\left(T_{I}\right)\right)=0$. Thus, from the analytic class number formula, we get

$$
\begin{aligned}
\left(e^{+} R_{0}: l\left(\mathcal{C}_{\beta}\right)\right) & =\left|\prod_{\chi \neq 1, \text { even }}(q-1) L_{k}(0, \bar{\chi}) \sum_{I \in \mathbb{P}_{\mathcal{S}},\left(F_{\chi}, M_{I}\right)=1} n_{I}\right| T_{I}\left|\chi(\beta(I)) \prod_{i \notin I}\left(1-\bar{\chi}\left(P_{i}\right)\right)\right| \\
& =(q-1)^{\left[F^{+}: k\right]-1} h\left(F^{+}\right) i_{\beta} .
\end{aligned}
$$

Since $h\left(\mathcal{O}_{F^{+}}\right)=h\left(F^{+}\right) / R\left(F^{+}\right)$, we complete the proof of Theorem.

When $\beta$ is multiplicative, the index $i_{\beta}$ becomes easy to compute.

THEOREM 3.2. If $\beta$ is multiplicative, then

$$
i_{\beta}=\left|\prod_{\chi \neq 1, \text { even }} \prod_{P_{i} \nmid F_{\chi}}\left(t_{i} \chi(\beta(i))+1-\bar{\chi}\left(P_{i}\right)\right)\right| .
$$

Proof: Note that $\left|T_{I}\right| n_{I}=\prod_{i \in I} t_{i}$ and $\chi(\beta(I))=\prod_{i \in I} \chi(\beta(i))$. Now we follow the proof of $[3$, Theorem 1.3] to prove the Theorem.

\section{A SYSTEM OF MAXIMAL INDEPENDENT UNITS}

In this section, we make a specific choice of $\beta$ to construct a maximal independent system of units. Define the function $\beta: \mathbb{P}_{S} \rightarrow \mathbb{Z}[G]$ as follows: $\beta$ is multiplicative and $\beta(i)=\nu_{i}$ for $i \in S$. Since $\lambda_{I} \in F_{M / M_{I}}$ and $\beta(I)$ is uniquely determined modulo $T_{I}=\operatorname{Gal}\left(F / F_{M / M_{I}}\right), \mathcal{C}_{\beta}$ is independent of the choice of $\mathcal{F}_{i}$. In the remainder of this paper, $\beta$ is fixed to this choice. 
THEOREM 4.1. With the above construction of $\mathcal{C}_{\beta}$, we have

$$
i_{\beta}=\prod_{i=1}^{s} t_{i}^{\left[G: J_{F} D_{i}\right]-1} f_{i}^{2\left[G: J_{F} D_{i}\right]-1}\left|J_{F} \cap D_{i} / J_{F} \cap T_{i}\right|^{-\left[G: J_{F} D_{i}\right]} .
$$

In particular, if $F$ is a real extension, then

$$
i_{\beta}=\prod_{i=1}^{s} t_{i}^{g_{i}-1} f_{i}^{2 g_{i}-1} .
$$

Proof: For $s=1$, we have both sides of the theorem equal to 1 . Suppose $s \geqslant 2$. Note that the condition $P_{i} \nmid F_{\chi}$ is equivalent to $T_{i} \subset \operatorname{ker} \chi$. If $\chi\left(P_{i}\right)=1$ (that is, $\left.D_{i} \subset \operatorname{ker} \chi\right)$, then $\chi(\beta(i))=f_{i}$. And the condition that $\chi \neq 1$ even, $P_{i} \nmid F_{\chi}, \chi\left(P_{i}\right)=1$ is equivalent to $J_{F} D_{i} \subset \operatorname{ker} \chi, \chi \neq 1$. Thus there are $\left[G: J_{F} D_{i}\right]-1$ such $\chi$.

If $\chi\left(P_{i}\right) \neq 1$ (that is, $D_{i} \not \subset$ ker $\chi$ ), $\chi(\beta(i))=0$. Thus for all $\chi \neq 1$ even with $P_{i} \nmid F_{\chi}$, $\chi\left(P_{i}\right) \neq 1, \bar{\chi}\left(P_{i}\right)$ ranges over all nontrivial $\left|D_{i} /\left(J_{F} \cap D_{i}\right) T_{i}\right|$-th roots of unity and it takes each values $\left[G: J_{F} D_{i}\right]$ times. Since $\left|D_{i} /\left(J_{F} \cap D_{i}\right) T_{i}\right|=f_{i}\left|J_{F} \cap D_{i} / J_{F} \cap T_{i}\right|^{-1}$, we get $i_{\beta}=\prod_{i=1}^{s}\left(\left(t_{i} f_{i}\right)^{\left[G: J_{F} D_{i}\right]-1}\right) \cdot\left(f_{i} /\left|J_{F} \cap D_{i} / J_{F} \cap T_{i}\right|\right)^{\left[G: J_{F} D_{i}\right]}$. So it completes the proof.

When $F$ is a real extension, we can make another subgroup of $\mathcal{O}_{F}^{*}$ which has smaller index than $\mathcal{C}_{\beta}$. If, for a monic divisor $N$ of $M, F_{N}$ is a real extension, then

$$
\begin{aligned}
N_{K_{N} / F_{N}}\left(\lambda_{N}\right)^{\sigma-1} & =N_{K_{N}^{+} / F_{N}}\left(N_{K_{N} / K_{N}^{+}}\left(\lambda_{N}^{\sigma-1}\right)\right) \\
& =N_{K_{N}^{+} / F_{N}}\left(\left(\lambda_{N}^{\sigma-1}\right)^{q-1}\right) \\
& =\left(N_{K_{N}^{+} / F_{N}}\left(\lambda_{N}^{\sigma-1}\right)\right)^{q-1}
\end{aligned}
$$

because $\lambda_{N}^{\sigma-1} \in K_{N}^{+}$. Since $F$ is real, for $\sigma \in G, \lambda(\beta)^{\sigma-1}=\varepsilon_{\sigma}^{q-1}$ with an explicit unit $\varepsilon_{\sigma} \in \mathcal{O}_{F}^{*}$. We define $\mathcal{C}_{\beta}^{\prime}$ as the subgroup of $\mathcal{O}_{F}^{*}$ generated by $\mathbb{F}_{q}^{*} \cup\left\{\varepsilon_{\sigma}: \sigma \in G, \sigma \neq 1\right\}$. Then it is easy to see that

COROLlaRY 4.2. When $F$ is a real extension, we have

$$
\left[\mathcal{O}_{F}^{*}: \mathcal{C}_{\beta}^{\prime}\right]=h\left(\mathcal{O}_{F}\right) \prod_{i=1}^{s} t_{i}^{g_{i}-1} f_{i}^{2 g_{i}-1}
$$

\section{NUMERICAL COMPARISON OF INDEXES}

In this section we compare the index $i_{\beta}$ with Feng-Yin's index ([2, Theorem 4]). For simplicity suppose $F=K_{M}^{+}$. Then we can simplify $i(\mathcal{D})$ in $[2$, Theorem 4] as

$$
i(\mathcal{D})=\prod_{i \in T} \frac{\left(\left(t_{i}+1\right)^{f_{i}}-1\right)^{g_{i}}}{t_{i}} \prod_{i \notin T} f_{i},
$$


for any subset $T$ of $S$ containing $T_{0}=\left\{i \in S: \chi\left(P_{i}\right)=1\right.$, for some $\left.\chi \neq 1\right\}$. When $T=S([2$, Theorem 5]), we have

$$
i(\mathcal{D})=\prod_{i=1}^{s} \frac{\left(\left(t_{i}+1\right)^{f_{i}}-1\right)^{g_{i}}}{t_{i}}
$$

which corresponds to the Ramachandra's unit system in a cyclotomic number field. We denote this index by $i_{R}$. As in Greither, it is easy to see that $i_{R} \geqslant i(\mathcal{D}) \geqslant i_{\beta}$. It is easy to see that $T_{0}$ is the set consisting of $i \in S$ such that $g_{i}>1$. Then $f_{i} g_{i}=\left[K_{\left.M / P_{i}^{c_{i}}: k\right]}\right.$ $=\Phi\left(M / P_{i}^{e_{i}}\right) /(q-1)$. Here $\Phi(M)$ denotes the order of $(\mathbb{A} / M \mathbb{A})^{*}$ for any $M \in \mathbb{A}$. Since $(q-1) \mid \Phi(P)$, if $s \geqslant 4, f_{i}$ can not have the order $\Phi\left(M / P_{i}^{e_{i}}\right) /(q-1)$ and so $T_{0}$ becomes $S$. Therefore $i(\mathcal{D})=i_{R}$ for $s \geqslant 4$. Now we show the numerical behaviour of the indexes $i_{R}, i(\mathcal{D})$, and $i_{\beta}$.

EXAMPLE 1. We consider an example with $q=3, s=3$ and $M=T\left(T^{2}+1\right)\left(T^{3}+T^{2}-1\right)$.

Then we get

$$
i_{R}=2^{12} \cdot 5^{4} \cdot 7 \cdot 41 \cdot 53^{3} \cdot 73 \cdot 4795973261^{3} \cdot 797161^{3} \cdot 398581^{3} \cdot 6481(84 \text { digits })
$$

with the optimal choice of $T=\{1\}$ and

$$
i(\mathcal{D})=2^{11} \cdot 5^{2} \cdot 13 \cdot 53^{2} \cdot 4795973261^{2} \cdot 797161^{2} \cdot 398581^{2}(52 \text { digits }) ;
$$

and finally

$$
i_{\beta}=2^{11} \cdot 13^{4} \text { (8 digits) }
$$

EXAMPLE 2. We take $s \geqslant 4$ and just compare $i_{R}$ and $i_{\beta}$. For $q=3$ and $M=T$ $(T-1)\left(T^{2}+1\right)\left(T^{3}+T^{2}-1\right)$, we get

$$
i_{R}=\frac{\left(3^{52}-1\right)^{4}}{2} \frac{\left(9^{26}-1\right)^{2}}{8} \frac{\left(27^{8}-1\right)^{2}}{26} \frac{\left(3^{104}-1\right)^{2}}{2}(269 \text { digits }) ;
$$

and

$$
i_{\beta}=2^{43} \cdot 13^{14} \text { (29 digits). }
$$

EXAMPLE 3. We take $q=3$ and $M=T(T+1)(T-1)\left(T^{2}+1\right)\left(T^{3}+T^{2}-1\right)$, then $i_{R}$ has 738 decimal digits and

$$
i_{\beta}=2^{125} \cdot 13^{39}(82 \text { digits })
$$

ExAmPLE 4. Finally, we take $q=3$ and $M=T(T+1)(T-1)\left(T^{2}+1\right)\left(T^{3}+T^{2}-1\right)$ $\left(T^{3}-T-1\right)$, then $i_{R}$ has 20440 decimal digits and

$$
i_{\beta}=2^{3105} \cdot 13^{1080}(2138 \text { digits }) .
$$

Note that with numbers of this size, it is really important to have an explicit prime factorisation of the index and the index $i_{\beta}$ is easy to factorise into primes. 


\section{R-CYCLIC SUBMODULE OF U}

Kučera ([5, Section 3]) constructed an $R$-cyclic submodule of the Sinnott's module $U$ using Greither's method in the number field case. In this section, we also construct an $R$-cyclic submodule of $U$ in the rational function field case. First we recall the definition of $U$. For $i \in S$, let $e_{P_{i}}=s\left(T_{i}\right) / t_{i}$ the idempotent associated to $P_{i}$. The Sinnott's module $U$ is defined as the $R$-submodule of $\mathbb{Q}[G]$ generated by $\left\{s\left(T_{I}\right) \prod_{i \notin I}\left(1-\mathcal{F}_{i}^{-1} e_{P_{i}}\right): I \subset S\right\}$. It is well known ([4, Proposition 2.1]) that $U$ is a free $\mathbb{Z}$-module of rank $|G|$. We put

$$
\begin{aligned}
g & =\sum_{I \subset S}\left(n_{I} \beta(I) s\left(T_{I}\right) \prod_{i \notin I}\left(1-\mathcal{F}_{i}^{-1} e_{P_{i}}\right)\right)=\sum_{I \subset S}\left(\prod_{i \in I} s\left(D_{i}\right)\right)\left(\prod_{i \notin I}\left(1-\mathcal{F}_{i}^{-1} e_{P_{i}}\right)\right) \\
& =\prod_{i=1}^{s}\left(s\left(D_{i}\right)+1-\mathcal{F}_{i}^{-1} e_{P_{i}}\right) \in U .
\end{aligned}
$$

Then $\chi(g)=\prod_{i=1}^{s}\left(\chi\left(s\left(D_{i}\right)\right)+1-\bar{\chi}\left(P_{i}\right) \chi\left(e_{P_{i}}\right)\right)$. Since the condition $\chi\left(e_{P_{i}}\right)=1$ is equivalent to $T_{i} \subset \operatorname{ker} \chi$, we have

$$
\chi(g)=\prod_{D_{i} \subset \operatorname{ker} \chi} t_{i} f_{i} \cdot \prod_{T_{i} \subset \mathrm{ker} \chi, D_{i} \subset \operatorname{cker} \chi}\left(1-\bar{\chi}\left(P_{i}\right)\right) \neq 0 .
$$

Let $e_{1}=s(G) /|G|$. Then it is easy to see that

$$
\left(1-e_{1}\right) l(\lambda(\beta))=\omega_{F}^{*} g,
$$

where $\omega_{F}^{*}=(q-1) \sum_{\chi \neq 1, \text { even }} L_{k}(0, \bar{\chi}) e_{\chi} \in \mathbb{Q}[G]$. We recall $e^{+}=s\left(J_{F}\right) / \delta_{F}$ and $e^{-}=1-e^{+}$.

Proposition 6.1. Let $z_{i}=\left|J_{F} \cap D_{i} / J_{F} \cap T_{i}\right|$ for $i \in S$.

(1) $\quad(R: g R)=\prod_{\chi} \chi(g)=\prod_{i=1}^{s} t_{i}^{g_{i}} f_{i}^{2 g_{i}}$.

(2) $\left(e^{+} R: g e^{+} R\right)=\prod_{\chi, \text { even }} \chi(g)=\prod_{i=1}^{s} t_{i}^{\left[G: J_{F} D_{i}\right]} f_{i}^{2\left[G: J_{F} D_{i}\right]} z_{i}{ }^{-\left[G: J_{F} D_{i}\right]}$.

(3) $\left(e^{-} R: g e^{-} R\right)=\prod_{\chi, o d d} \chi(g)=\prod_{i=1}^{s} t_{i}^{\left(g_{i}-\left[G: J_{F} D_{i}\right]\right)} f_{i}^{2\left(g_{i}-\left[G: J_{F} D_{i}\right]\right)} z_{i}{ }^{\left[G: J_{F} D_{i}\right]}$.

Proof: (1) is easily proved as Greither ([3, Proof of Proposition 2.1]). The proof of (2) is almost same as the proof of Theorem 4.1 and (3) follows from (1) and (2).

\section{REFERENCES}

[1] S. Bae. H. Jung and J. Ahn, 'Cyclotomic units and Stickelberger ideals of global function fields', (preprint).

[2] K. Feng and L. Yin, 'Maximal independent system of units in cyclotomic function fields', Sci. China Ser. A. 34 (1991), 908-919.

[3] C. Greither, 'Improving Ramachandra's and Levesque's unit index', in Number theory (Ottawa, ON, 1996) (Amer. Math. Soc., Providence, RI), pp. 111-120. 
[4] F. Harrop, 'Circular units of function fields', Trans. Amer. Math. Soc. 341 (1994), 405-421.

[5] R. Kučera, 'A generalization of a unit index of Greither', Acta Math. Inform. Univ. Ostraviensis 6 (1998), 149-154.

[6] C. Levesque, 'On improving Ramachadra's unit index', in Number theory (Banff, Alberta) 1988 (W. de Gruyter, Berlin, 1990), pp. 325-338.

[7] K. Ramachadra, 'On the units of cyclotomic fields', Acta Arith. XII (1966), 165-173.

[8] L. Washington, Introduction to cyclotomic fields (Springer-Verlag, Berlin, Heidelberg, New York, 1997).

Department of Mathematics

Korea University

Seoul

Korea 136-701

e-mail: hyjung@mathx.kaist.ac.kr
Department of Mathematics

KAIST

Taejon

Korea 305-701

e-mail: jaehyun@mathx.kaist.ac.kr 